\title{
Completion versus removal of redundancy by perturbation
}

\author{
Ole Christensen, Marzieh Hasannasab
}

June 2, 2021

\begin{abstract}
A sequence $\left\{g_{k}\right\}_{k=1}^{\infty}$ in a Hilbert space $\mathcal{H}$ has the expansion property if each $f \in \overline{\operatorname{span}}\left\{g_{k}\right\}_{k=1}^{\infty}$ has a representation $f=\sum_{k=1}^{\infty} c_{k} g_{k}$ for some scalar coefficients $c_{k}$. In this paper we analyze the question whether there exist small norm-perturbations of $\left\{g_{k}\right\}_{k=1}^{\infty}$ which allow to represent all $f \in \mathcal{H}$; the answer turns out to be yes for frame sequences and Riesz sequences, but no for general basic sequences. The insight gained from the analysis is used to address a somewhat dual question, namely, whether it is possible to remove redundancy from a sequence with the expansion property via small norm-perturbations; we prove that the answer is yes for frames $\left\{g_{k}\right\}_{k=1}^{\infty}$ such that $g_{k} \rightarrow 0$ as $k \rightarrow \infty$, as well as for frames with finite excess. This particular question is motivated by recent progress in dynamical sampling.
\end{abstract}

Keywords Frames, Riesz bases, completeness, redundancy 2000 Mathematics Subject Classification: 42C40

\section{Introduction}

Let $\mathcal{H}$ denote a separable infinite-dimensional Hilbert space and suppose that a given sequence $\left\{g_{k}\right\}_{k=1}^{\infty}$ in $\mathcal{H}$ has the expansion property, i.e., that each $f \in \overline{\operatorname{span}}\left\{g_{k}\right\}_{k=1}^{\infty}$ has a representation

$$
f=\sum_{k=1}^{\infty} c_{k} g_{k}
$$


for certain coefficients $c_{k} \in \mathbb{C}$. Our goal is to address the following question: when and how can we perform small norm-perturbations on the sequence $\left\{g_{k}\right\}_{k=1}^{\infty}$ and hereby obtain a sequence $\left\{\psi_{k}\right\}_{k=1}^{\infty}$ such that arbitrary elements $f \in \mathcal{H}$ have an expansion $f=\sum_{k=1}^{\infty} c_{k} \psi_{k}$ for certain coefficients $c_{k} \in \mathbb{C}$ ?

Formulated as above, the question is clearly a completion problem. We will show that the completion problem has an affirmative answer for the so-called Riesz sequences and frame sequences, but not for general basic sequences; along the way we also consider a number of other completion problems. Interestingly, the insight gained from the above analysis can be used to address a somewhat dual question: when and how can a redundant system $\left\{g_{k}\right\}_{k=1}^{\infty}$ be turned into a complete but nonredundant system $\left\{\psi_{k}\right\}_{k=1}^{\infty}$ by small norm-perturbations? We will provide a positive answer to this question for a number of frames, in particular, for the so-called near-Riesz bases introduced by Holub in [13]. Additional motivation for this particular question will be provided at the end of the paper.

The paper is organized as follows. In the rest of the introduction we set the stage by providing a number of definitions and results from the literature. In Section 2 we present the results about the completion problem; the dual problem concerning removal of redundancy is considered in Section 3 ,

A sequence $\left\{g_{k}\right\}_{k=1}^{\infty}$ in the Hilbert space $\mathcal{H}$ is called a frame for $\mathcal{H}$ if there exist constants $A, B>0$ such that

$$
A\|f\|^{2} \leq \sum_{k=1}^{\infty}\left|\left\langle f, g_{k}\right\rangle\right|^{2} \leq B\|f\|^{2}, \forall f \in \mathcal{H}
$$

suitable numbers $A, B$ are called lower, resp. upper frame bounds. The sequence $\left\{g_{k}\right\}_{k=1}^{\infty}$ is called a Bessel sequence if at least the right-hand inequality in (1.2) holds. A frame which is at the same time a basis, is called a Riesz basis. Note that several other characterizations of frames and Riesz bases exist, e.g., in terms of operator theory. For example, if $\left\{e_{k}\right\}_{k=1}^{\infty}$ is a given orthonormal basis for $\mathcal{H}$, frames for $\mathcal{H}$ are precisely the sequences $\left\{V e_{k}\right\}_{k=1}^{\infty}$ where $V: \mathcal{H} \rightarrow \mathcal{H}$ is a bounded surjective operator; Riesz bases correspond precisely to the case where the operator $V$ also is injective. Finally, a sequence $\left\{g_{k}\right\}_{k=1}^{\infty}$ which is a frame for the (sub)space $\mathcal{K}:=\overline{\operatorname{span}}\left\{g_{k}\right\}_{k=1}^{\infty}$, is called a frame sequence; Riesz sequences are defined in the analogue way.

One of the key reasons for the interest in frames is that a frame has the expansion property: in fact, given any frame $\left\{g_{k}\right\}_{k=1}^{\infty}$, there exists a so-called 
dual frame $\left\{f_{k}\right\}_{k=1}^{\infty}$ such that

$$
f=\sum_{k=1}^{\infty}\left\langle f, f_{k}\right\rangle g_{k}, \forall f \in \mathcal{H}
$$

In general, the dual frame $\left\{f_{k}\right\}_{k=1}^{\infty}$ is not unique: indeed, the case where $\left\{g_{k}\right\}_{k=1}^{\infty}$ is a Riesz basis is characterized precisely by the existence of a unique dual. We refer to [9] for more information about frames and Riesz bases, also about their history and applications.

The following Lemma collects a number of well-known results concerning norm-perturbations of various sequences with the expansion property.

Lemma 1.1 Consider two sequences $\left\{g_{k}\right\}_{k=1}^{\infty},\left\{h_{k}\right\}_{k=1}^{\infty}$ in $\mathcal{H}$, satisfying that

$$
\sum_{k=1}^{\infty}\left\|g_{k}-h_{k}\right\|^{2}<A,
$$

for a value of $A$ as specified below. Then the following holds:

(i) [7] If $\left\{g_{k}\right\}_{k=1}^{\infty}$ is a frame for $\mathcal{H}$ with lower bound $A$, then $\left\{h_{k}\right\}_{k=1}^{\infty}$ is a frame for $\mathcal{H}$.

(ii) [8] If $\left\{g_{k}\right\}_{k=1}^{\infty}$ is a Riesz sequence with lower bound $A$, then $\left\{h_{k}\right\}_{k=1}^{\infty}$ is a Riesz sequence; furthermore, codim $\left(\overline{\operatorname{span}}\left\{g_{k}\right\}_{k=1}^{\infty}\right)=\operatorname{codim}\left(\overline{\operatorname{span}}\left\{h_{k}\right\}_{k=1}^{\infty}\right)$.

Alternative norm-perturbation conditions are formulated in [6]; however, they need that we have access to information about a dual frame, which is not the case in the current paper. Note also that a number of classical results about norm-perturbation (typically for orthonormal sequences) are collected in [17]. Observe that more general perturbation results are available in the literature, typically formulated in terms of certain operators rather than norm-perturbations; see, e.g., [9] and the references therein.

\section{Completion via norm-perturbation}

Our main interest is to consider the completion problem for sequences $\left\{g_{k}\right\}_{k=1}^{\infty}$ having the expansion property. However, we first state a number of other completion properties, some of which will be needed in later proofs. Given 
any sequence $\left\{g_{k}\right\}_{k=1}^{\infty}$ in $\mathcal{H}$, we define its excess $\mathcal{E}\left(\left\{g_{k}\right\}_{k=1}^{\infty}\right)$ as the maximal number of elements that can be removed without changing the spanned space, i.e.,

$$
\mathcal{E}\left(\left\{g_{k}\right\}_{k=1}^{\infty}\right):=\max \sharp\left\{J \subset \mathbb{N} \mid \overline{\operatorname{span}}\left\{g_{k}\right\}_{k \in \mathbb{N} \backslash J}=\overline{\operatorname{span}}\left\{g_{k}\right\}_{k=1}^{\infty}\right\} .
$$

Proposition 2.1 Let $\left\{g_{k}\right\}_{k=1}^{\infty}$ be a sequence in $\mathcal{H}$. Then the following hold:

(i) If $\left\{g_{k}\right\}_{k=1}^{\infty}$ is not norm-bounded below, there exists a complete sequence $\left\{\psi_{k}\right\}_{k=1}^{\infty}$ such that

$$
\left\|g_{k}-\psi_{k}\right\| \rightarrow 0 \text { as } k \rightarrow \infty
$$

(ii) If $\mathcal{E}\left(\left\{g_{k}\right\}_{k=1}^{\infty}\right) \geq \operatorname{codim}\left(\overline{\operatorname{span}}\left\{g_{k}\right\}_{k=1}^{\infty}\right)$, there exists a complete sequence $\left\{\psi_{k}\right\}_{k=1}^{\infty}$ such that (2.2) holds.

(iii) If $\left\{g_{k}\right\}_{k=1}^{\infty}$ is convergent, there exists a complete sequence $\left\{\psi_{k}\right\}_{k=1}^{\infty}$ such that (2.2) holds; in particular, $\left\{\psi_{k}\right\}_{k=1}^{\infty}$ converges to the same limit as $\left\{g_{k}\right\}_{k=1}^{\infty}$.

In all the stated cases, given any $\delta>0$, the sequence $\left\{\psi_{k}\right\}_{k=1}^{\infty}$ can be chosen such that $\left\|g_{k}-\psi_{k}\right\| \leq \delta$ for all $k \in \mathbb{N}$.

Proof. For the proof of (i), given $\delta>0$, choose a frame $\left\{f_{k}\right\}_{k=1}^{\infty}$ for $\mathcal{H}$ such that $\left\|f_{k}\right\| \leq \delta$ for all $k \in \mathbb{N}$ and $\left\|f_{k}\right\| \rightarrow 0$ as $k \rightarrow \infty$; for example, letting $\left\{e_{k}\right\}_{k=1}^{\infty}$ denote any orthonormal basis, we can take

$$
\left\{f_{k}\right\}_{k=1}^{\infty}=\left\{\delta e_{1}, \frac{\delta}{\sqrt{2}} e_{2}, \frac{\delta}{\sqrt{2}} e_{2}, \frac{\delta}{\sqrt{3}} e_{3}, \frac{\delta}{\sqrt{3}} e_{3}, \frac{\delta}{\sqrt{3}} e_{3}, \ldots\right\} .
$$

Denote the lower frame bound for the frame $\left\{f_{k}\right\}_{k=1}^{\infty}$ by $A$. Choose now a subsequence $\left\{g_{k_{n}}\right\}_{n=1}^{\infty}$ of $\left\{g_{k}\right\}_{k=1}^{\infty}$ such that $\left\|g_{k_{n}}\right\|^{2} \leq \frac{3 A}{\pi^{2} n^{2}}, n \in \mathbb{N}$; then

$$
\sum_{n=1}^{\infty}\left\|f_{n}-\left(f_{n}+g_{k_{n}}\right)\right\|^{2}=\sum_{n=1}^{\infty}\left\|g_{k_{n}}\right\|^{2} \leq \frac{A}{2} .
$$

Using Lemma1.1(i), this implies that $\left\{f_{n}+g_{k_{n}}\right\}_{n=1}^{\infty}$ is a frame for $\mathcal{H}$ and hence complete. Thus, the sequence $\left\{\psi_{k}\right\}_{k=1}^{\infty}$ formed from $\left\{g_{k}\right\}_{k=1}^{\infty}$ by replacing the elements $\left\{g_{k_{n}}\right\}_{n=1}^{\infty}$ by $\left\{f_{n}+g_{k_{n}}\right\}_{n=1}^{\infty}$ will satisfy the requirements. 
For the proof of (ii) we first assume additionally that $M:=\operatorname{codim}\left(\overline{\operatorname{span}}\left\{g_{k}\right\}_{k=1}^{\infty}\right)$ is finite. Without loss of generality and only for notational convenience, assume that the sequence $\left\{g_{k}\right\}_{k=1}^{\infty}$ is ordered such that $g_{1}, \ldots, g_{M} \in \overline{\operatorname{span}}\left\{g_{k}\right\}_{k=M+1}^{\infty}$, and take an orthonormal basis $\left\{e_{k}\right\}_{k=1}^{M}$ for the orthogonal complement $\left(\overline{\operatorname{span}}\left\{g_{k}\right\}_{k=1}^{\infty}\right)^{\perp}$. Then the sequence

$$
\left\{\psi_{k}\right\}_{k=1}^{\infty}=\left\{g_{1}+\delta e_{1}, g_{2}+\frac{\delta}{2} e_{2}, \ldots, g_{M}+\frac{\delta}{M} e_{M}, g_{M+1}, g_{M+2}, \ldots\right\}
$$

satisfies the requirements. The case where $\mathcal{E}\left(\left\{g_{k}\right\}_{k=1}^{\infty}\right)=\operatorname{codim}\left(\overline{\operatorname{span}}\left\{g_{k}\right\}_{k=1}^{\infty}\right)=$ $\infty$ is similar and only requires minor notational modifications.

For the proof of (iii), assume that the sequence $\left\{g_{k}\right\}_{k=1}^{\infty}$ converges to $f \in \mathcal{H}$. Given $\delta>0$, choose $K \in \mathbb{N}$ such that $\left\|f-g_{k}\right\| \leq \delta / 2$ for $k \geq K$. Let $\left\{e_{k}\right\}_{k=1}^{\infty}$ denote an orthonormal basis for $\mathcal{H}$ and define $\left\{\psi_{k}\right\}_{k=1}^{\infty}$ by

$$
\psi_{k}:= \begin{cases}g_{k}, & \text { if } k=1, \ldots, K-1 \\ f, & \text { if } k=K \\ f+\frac{\delta}{2^{k-K}} e_{k-K}, & \text { if } k>K .\end{cases}
$$

Then $\operatorname{span}\left\{e_{k}\right\}_{k=1}^{\infty} \subseteq \operatorname{span}\left\{\psi_{k}\right\}_{k=1}^{\infty}$, so $\operatorname{span}\left\{\psi_{k}\right\}_{k=1}^{\infty}$ is clearly complete. Furthermore, for $k \geq K$,

$$
\left\|g_{k}-\psi_{k}\right\| \leq\left\|g_{k}-f\right\|+\left\|f-\psi_{k}\right\| \leq \delta
$$

and $\left\|g_{k}-\psi_{k}\right\| \rightarrow 0$ as $k \rightarrow \infty$.

We are now ready to consider the completion problem for Riesz sequences and frame sequences. The proofs rely on an interesting result proved recently by V. Olevskii:

Lemma 2.2 [16, 15] If $\left\{e_{k}\right\}_{k=1}^{\infty}$ is an orthonormal sequence in $\mathcal{H}$, there exists an orthonormal basis $\left\{\chi_{k}\right\}_{k=1}^{\infty}$ for $\mathcal{H}$ such that

$$
\left\|e_{k}-\chi_{k}\right\| \rightarrow 0 \text { as } k \rightarrow \infty \text {. }
$$

In addition, given any $\delta>0$, the sequence $\left\{\chi_{k}\right\}_{k=1}^{\infty}$ can be chosen such that $\left\|e_{k}-\chi_{k}\right\| \leq \delta$ for all $k \in \mathbb{N}$. 
Theorem 2.3 Let $\left\{g_{k}\right\}_{k=1}^{\infty}$ be a sequence in $\mathcal{H}$. Then the following hold:

(i) If $\left\{g_{k}\right\}_{k=1}^{\infty}$ is a Riesz sequence, there exists a Riesz basis $\left\{\psi_{k}\right\}_{k=1}^{\infty}$ for $\mathcal{H}$ such that

$$
\left\|g_{k}-\psi_{k}\right\| \rightarrow 0 \text { as } k \rightarrow \infty
$$

(ii) If $\left\{g_{k}\right\}_{k=1}^{\infty}$ is a frame sequence, there exists a frame $\left\{\psi_{k}\right\}_{k=1}^{\infty}$ for $\mathcal{H}$ such that (2.3) holds.

(iii) If $\left\{g_{k}\right\}_{k=1}^{\infty}$ is a Bessel sequence, there exists a complete Bessel sequence $\left\{\psi_{k}\right\}_{k=1}^{\infty}$ such that (2.3) holds.

In all the stated cases, given any $\delta>0$, the sequence $\left\{\psi_{k}\right\}_{k=1}^{\infty}$ can be chosen such that $\left\|g_{k}-\psi_{k}\right\| \leq \delta$ for all $k \in \mathbb{N}$.

Proof. We first prove (iii). Thus, let $\left\{g_{k}\right\}_{k=1}^{\infty}$ be a Bessel sequence in $\mathcal{H}$, and let $\mathcal{K}:=\overline{\operatorname{span}}\left\{g_{k}\right\}_{k=1}^{\infty}$; we can assume that $\mathcal{K}^{\perp} \neq\{0\}$. Also, if $\mathcal{K}$ is finite-dimensional, the results follow from Proposition 2.1 (ii), so we will assume that $\mathcal{K}$ is infinite-dimensional. Now, by the standard properties of a Bessel sequence [9], choose an orthonormal basis $\left\{e_{k}\right\}_{k=1}^{\infty}$ for $\mathcal{K}$ and a bounded operator $U: \mathcal{K} \rightarrow \mathcal{K}$ such that $g_{k}=U e_{k}, k \in \mathbb{N}$. Associated with the orthonormal sequence $\left\{e_{k}\right\}_{k=1}^{\infty}$, choose the orthonormal basis $\left\{\chi_{k}\right\}_{k=1}^{\infty}$ for $\mathcal{H}$ as in Lemma 2.2, and define a bounded operator $V: \mathcal{H} \rightarrow \mathcal{H}$ by

$$
V=U \text { on } \mathcal{K}, V=I \text { on } \mathcal{K}^{\perp} .
$$

Since the range of the operator $U$ contains the vectors $\left\{g_{k}\right\}_{k=1}^{\infty}$, it is dense in $\mathcal{K}$. Thus, the range of the operator $V$ is dense in $\mathcal{H}$; this implies that the sequence $\left\{\psi_{k}\right\}_{k=1}^{\infty}:=\left\{V \chi_{k}\right\}_{k=1}^{\infty}$ is complete in $\mathcal{H}$. A direct calculation reveals that $\left\{\psi_{k}\right\}_{k=1}^{\infty}$ is a Bessel sequence. Furthermore, for all $k \in \mathbb{N}$,

$$
\left\|g_{k}-\psi_{k}\right\|=\left\|U e_{k}-V \chi_{k}\right\|=\left\|V e_{k}-V \chi_{k}\right\| \leq\|V\|\left\|e_{k}-\chi_{k}\right\| .
$$

Since the operator $V$ only depends on the sequence $\left\{g_{k}\right\}_{k=1}^{\infty}$ (and the fixed choice of $\left\{e_{k}\right\}_{k=1}^{\infty}$ ), this proves (iii). This also gives the proof of (i)-(ii). Indeed, if $\left\{g_{k}\right\}_{k=1}^{\infty}$ is a frame sequence, the range of the operator $U$ equals $\mathcal{K}$, which implies that the range of the operator $V$ equals $\mathcal{H}$, and hence $\left\{\psi_{k}\right\}_{k=1}^{\infty}$ is a frame for $\mathcal{H}$; and if $\left\{f_{k}\right\}_{k=1}^{\infty}$ is a Riesz sequence, the operator $U: \mathcal{K} \rightarrow \mathcal{K}$ is bijective, implying that $V: \mathcal{H} \rightarrow \mathcal{H}$ is bijective, and hence that $\left\{\psi_{k}\right\}_{k=1}^{\infty}$ is a Riesz sequence. 
Remark 2.4 Despite the fact that $\delta>0$ can be chosen arbitrarily small in Theorem 2.3, there is a restriction on how "close" the sequence $\left\{\psi_{k}\right\}_{k=1}^{\infty}$ can be to the sequence $\left\{g_{k}\right\}_{k=1}^{\infty}$. Indeed, if $\left\{g_{k}\right\}_{k=1}^{\infty}$ is a (non-complete) Riesz sequence with lower bound $A$, then the sequence $\left\{\psi_{k}\right\}_{k=1}^{\infty}$ in Theorem 2.3)(i) must satisfy that

$$
\sum_{k=1}^{\infty}\left\|g_{k}-\psi_{k}\right\|^{2} \geq A
$$

otherwise Lemma 1.1(ii) would imply that $\left\{\psi_{k}\right\}_{k=1}^{\infty}$ is non-complete as well. A similar result holds for frame sequences, although the lower bound on the infinite sum in (2.5) will involve the gap between two particular subspaces of $\mathcal{H}$; see [8, 10] for more detailed information.

Theorem 2.3 makes it natural to ask whether a basic sequence (i.e., a Schauder basis for a subspace) also can be extended to a Schauder basis for $\mathcal{H}$ by small norm-perturbations of the elements. The following example shows that the answer is no in general, unless additional assumptions are added.

Example 2.5 Let $\left\{e_{k}\right\}_{k=1}^{\infty}$ denote an orthonormal basis for $\mathcal{H}$ and consider the sequence

$$
\left\{g_{k}\right\}_{k=1}^{\infty}=\left\{2 e_{2}, 4 e_{4}, 6 e_{6}, \ldots\right\}=\left\{2 k e_{2 k}\right\}_{k=1}^{\infty} .
$$

Clearly $\left\{g_{k}\right\}_{k=1}^{\infty}$ is a basic sequence. Now, given any $\left.\delta \in\right] 0,2 \sqrt{6} \pi^{-1}$ [, consider a sequence $\left\{\psi_{k}\right\}_{k=1}^{\infty}$ in $\mathcal{H}$ such that $\left\|g_{k}-\psi_{k}\right\| \leq \delta$ for all $k \in \mathbb{N}$. Then

$$
\sum_{k=1}^{\infty}\left\|e_{2 k}-\frac{1}{2 k} \psi_{k}\right\|^{2}=\sum_{k=1}^{\infty} \frac{1}{4 k^{2}}\left\|g_{k}-\psi_{k}\right\|^{2} \leq \frac{\pi^{2} \delta^{2}}{24}<1 .
$$

Since $\left\{e_{2 k}\right\}_{k=1}^{\infty}$ forms a Riesz sequence with lower bound $A=1$, Lemma1.1(ii) implies that $\left\{(2 k)^{-1} \psi_{k}\right\}_{k=1}^{\infty}$ also forms a Riesz sequence, spanning a space of the same codimension as $\left\{e_{2 k}\right\}_{k=1}^{\infty}$; in particular, $\left\{\psi_{k}\right\}_{k=1}^{\infty}$ can not be complete in $\mathcal{H}$, and hence is not a Schauder basis for $\mathcal{H}$. 


\section{Removal of redundancy via norm-perturbations}

In this section the focus is on sequences $\left\{g_{k}\right\}_{k=1}^{\infty}$ having the expansion property on the entire underlying Hilbert space $\mathcal{H}$. Such expansions might be redundant, i.e., a given $f \in \mathcal{H}$ might have expansions $f=\sum_{k=1}^{\infty} c_{k} g_{k}$ for more than one choice of the scalar coefficients $\left\{c_{k}\right\}_{k=1}^{\infty}$. A typical example of a redundant sequence is a frame $\left\{g_{k}\right\}_{k=1}^{\infty}$ which is not a Riesz basis. Our goal

is to show that for certain frames $\left\{g_{k}\right\}_{k=1}^{\infty}$ the redundancy can be removed via small norm-perturbations of the vectors $g_{k}$.

Our first observation, stated next, does not even need the frame assumption or any other expansion property.

Theorem 3.1 Consider any sequence $\left\{g_{k}\right\}_{k=1}^{\infty}$ in $\mathcal{H}$ such that $g_{k} \rightarrow 0$ as $k \rightarrow \infty$. Then, given any $\delta>0$, there exists a Riesz basis $\left\{\psi_{k}\right\}_{k=1}^{\infty}$ for $\mathcal{H}$ such that

$$
\left\|g_{k}-\psi_{k}\right\| \leq \delta, \forall k \in \mathbb{N} \text {. }
$$

Proof. First, given any $\delta>0$, choose $K \in \mathbb{N}$ such that $\left\|g_{k}\right\|<\delta / 2$ for $k \geq K$. We will now construct $\left\{\psi_{k}\right\}_{k=1}^{\infty}$ recursively, of the form $\psi_{k}:=g_{k}+\varphi_{k}$ with the vectors $\varphi_{k}$ chosen as described next. First, take $\varphi_{1} \in \mathcal{H}$ such that $\left\|\varphi_{1}\right\| \leq \delta$ and $\psi_{1} \neq 0$. Then choose $\varphi_{2} \in \mathcal{H}$ such that $\left\|\varphi_{2}\right\| \leq \delta$ and $\left\{\psi_{1}, \psi_{2}\right\}$ is linearly independent. Continuing recursively, we finally choose $\varphi_{K} \in \mathcal{H}$ such that $\left\|\varphi_{K}\right\| \leq \delta$ and $\left\{\psi_{1}, \psi_{2}, \ldots, \psi_{K}\right\}$ is linearly independent. Then $\left\{\psi_{1}, \psi_{2}, \ldots, \psi_{K}\right\}$ is a Riesz basis for the subspace $V:=\operatorname{span}\left\{\psi_{1}, \psi_{2}, \ldots, \psi_{K}\right\}$. Now choose an orthonormal basis $\left\{e_{k}\right\}_{k=1}^{\infty}$ for $V^{\perp}$ and define $\psi_{k}$ for $k>K$ by $\psi_{k}:=\frac{\delta}{2} e_{k}$. Then $\left\{\psi_{k}\right\}_{k=1}^{\infty}$ is a Riesz basis for $\mathcal{H}$ and $\left\|g_{k}-\psi_{k}\right\| \leq \delta$ for all $k \in \mathbb{N}$.

The result in Theorem 3.1 immediately applies to a number of well-known frames in the literature:

Example 3.2 We state a number of examples of frames $\left\{g_{k}\right\}_{k=1}^{\infty}$ such that $g_{k} \rightarrow 0$ as $k \rightarrow \infty$ :

(i) Given any orthonormal basis $\left\{e_{k}\right\}_{k=1}^{\infty}$ for $\mathcal{H}$, the family

$$
\left\{g_{k}\right\}_{k=1}^{\infty}:=\left\{e_{1}, \frac{1}{\sqrt{2}} e_{2}, \frac{1}{\sqrt{2}} e_{2}, \frac{1}{\sqrt{3}} e_{3}, \frac{1}{\sqrt{3}} e_{3}, \frac{1}{\sqrt{3}} e_{3}, \ldots\right\}
$$

is a frame for $\mathcal{H}$. Clearly $g_{k} \rightarrow 0$ as $k \rightarrow \infty$. Note that this particular frame was used in the proof of Proposition 2.1. 
(ii) Let again $\left\{e_{k}\right\}_{k=1}^{\infty}$ be an orthonormal basis for $\mathcal{H}$ and fix any $\left.\alpha \in\right] 0,1[$. Let $\lambda_{\ell}:=1-\alpha^{-\ell}$ for $\ell \in \mathbb{N}$, and define the vectors

$$
g_{k}:=\sum_{\ell=1}^{\infty} \lambda_{\ell}^{k} \sqrt{1-\lambda_{\ell}^{2}} e_{\ell}, k \in \mathbb{N} .
$$

Then $\left\{g_{k}\right\}_{k=1}^{\infty}$ is a frame (the so-called Carleson frame), a result proved by Aldroubi et al. in [1, 2]. It is easy to see that $g_{k} \rightarrow 0$ as $k \rightarrow \infty$. Note that $\left\{g_{k}\right\}_{k=1}^{\infty}$ is heavily redundant: it can be proved that for any $N \in \mathbb{N}$, any subfamily $\left\{g_{N k}\right\}_{k \in \mathbb{N}}$ of $\left\{g_{k}\right\}_{k=1}^{\infty}$ is a redundant frame as well. From this point of view it is surprising that $\left\{g_{k}\right\}_{k=1}^{\infty}$ can be approximated by a Riesz basis, as stated in Theorem 3.1.

(iii) More generally than (ii), it was proved in [12] that any redundant frame that can be represented as an operator orbit $\left\{g_{k}\right\}_{k=1}^{\infty}=\left\{T^{k} \varphi\right\}_{k=1}^{\infty}$ for a bounded operator $T: \mathcal{H} \rightarrow \mathcal{H}$ and some $\varphi \in \mathcal{H}$ will have the property that $g_{k} \rightarrow 0$ as $k \rightarrow \infty$.

In order to reach the next result we need the following Lemma. Recall that the deficit of a sequence $\left\{g_{k}\right\}_{k=1}^{\infty}$ is defined as the codimension of the vector space $\overline{\operatorname{span}}\left\{g_{k}\right\}_{k=1}^{\infty}$.

Lemma 3.3 Let $\left\{e_{k}\right\}_{k=1}^{\infty}$ be an orthonormal basis for $\mathcal{H}$. Given any $\delta>0$ and any $N \in \mathbb{N}$, there exists an orthonormal system $\left\{\varepsilon_{k}\right\}_{k=1}^{\infty}$ with deficit $N$ such that $\left\|e_{k}-\varepsilon_{k}\right\| \leq \delta$ for all $k \in \mathbb{N}$.

Proof. Take any orthonormal system $\left\{\varphi_{k}\right\}_{k=1}^{\infty}$ with deficit $N$, and choose via Lemma 2.2 an orthonormal basis $\left\{\chi_{k}\right\}_{k=1}^{\infty}$ for $\mathcal{H}$ such that $\left\|\varphi_{k}-\chi_{k}\right\| \leq \delta$ for all $k \in \mathbb{N}$. Then, choose the unitary operator $U: \mathcal{H} \rightarrow \mathcal{H}$ such that $e_{k}=U \chi_{k}$, and let $\varepsilon_{k}:=U \varphi_{k}, k \in \mathbb{N}$. Then $\left\{\varepsilon_{k}\right\}_{k=1}^{\infty}$ is an orthonormal system with deficit $N$, and $\left\|e_{k}-\varepsilon_{k}\right\|=\left\|U \chi_{k}-U \varphi_{k}\right\|=\left\|\chi_{k}-\varphi_{k}\right\| \leq \delta$ for all $k \in \mathbb{N}$, as desired.

Theorem 3.4 Consider a frame of the form $\left\{g_{k}\right\}_{k=1}^{\infty}=\left\{g_{k}\right\}_{k=1}^{N} \cup\left\{g_{k}\right\}_{k=N+1}^{\infty}$, where $N \in \mathbb{N}$ and $\left\{g_{k}\right\}_{k=N+1}^{\infty}$ is a Riesz basis for $\mathcal{H}$. Then, given any $\delta>0$, there exists a Riesz basis $\left\{\psi_{k}\right\}_{k=1}^{\infty}$ such that $\left\|g_{k}-\psi_{k}\right\| \leq \delta$ for all $k \in \mathbb{N}$. 
Proof. First, consider an orthonormal basis for $\mathcal{H}$ indexed as $\left\{e_{k}\right\}_{k=N+1}^{\infty}$ and choose the bounded bijective operator $V: \mathcal{H} \rightarrow \mathcal{H}$ such that $g_{k}=V e_{k}$ for $k=$ $N+1, N+2, \ldots$ Using Lemma 3.3, choose an orthonormal system $\left\{\varepsilon_{k}\right\}_{k=N+1}^{\infty}$ with deficit $N$ such that $\left\|e_{k}-\varepsilon_{k}\right\| \leq \delta /\|V\|$ for $k=N+1, N+2, \ldots$. Then, letting $\psi_{k}:=V \varepsilon_{k}, k=N+1, N+2, \ldots$, the family $\left\{\psi_{k}\right\}_{k=N+1}^{\infty}$ is a Riesz sequence with deficit $N$, and $\left\|g_{k}-\psi_{k}\right\|=\left\|V e_{k}-V \varepsilon_{k}\right\| \leq \delta$ for $k=N+1, N+2, \ldots$

Now, consider the vector $g_{N}$. If $g_{N} \notin \overline{\operatorname{span}}\left\{\psi_{k}\right\}_{k=N+1}^{\infty}$, let $\psi_{N}:=g_{N}$; then $\left\{\psi_{k}\right\}_{k=N}^{\infty}$ is a Riesz sequence with deficit $N-1$. On the other hand, if $g_{N} \in \overline{\operatorname{span}}\left\{\psi_{k}\right\}_{k=N+1}^{\infty}$, choose any normalized vector $\varphi_{N} \notin \overline{\operatorname{span}}\left\{\psi_{k}\right\}_{k=N+1}^{\infty}$, and let $\psi_{N}:=g_{N}+\delta \varphi_{N}$; then again $\left\{\psi_{k}\right\}_{k=N}^{\infty}$ is a Riesz sequence with deficit $N-1$, and $\left\|g_{k}-\psi_{k}\right\| \leq \delta$ for $k=N, N+1, N+2, \ldots$ Applying now the same procedure on $g_{N-1}, g_{N-2}, \ldots, g_{1}$, we arrive at the desired Riesz basis $\left\{\psi_{k}\right\}_{k=1}^{\infty}$ in a finite number of steps.

Interestingly, frames of the type considered in Theorem 3.4 were called near-Riesz bases by Holub in the paper [13]; the above result provides an additional reason for this name being very appropriate.

Remark 3.5 Despite the fact that $\delta>0$ can be chosen arbitrarily small in Theorem 3.4, the Riesz basis $\left\{\psi_{k}\right\}_{k=1}^{\infty}$ must satisfy that $\sum_{k=1}^{\infty}\left\|g_{k}-\psi_{k}\right\|^{2} \geq A$, where $A$ is the lower frame bound for $\left\{g_{k}\right\}_{k=1}^{\infty}$; otherwise the results in [5] show that $\left\{\psi_{k}\right\}_{k=1}^{\infty}$ would be a frame with the same excess as $\left\{g_{k}\right\}_{k=1}^{\infty}$.

We want to point out that the proof of Theorem 3.4 somewhat hides the fact that it is highly nontrivial to get direct access to the Riesz basis $\left\{\psi_{k}\right\}_{k=1}^{\infty}$, especially due to the intriguing and deep construction by V. Olevskii playing a key role in the argument. The next example illustrates this by a concrete construction.

Example 3.6 Let again $\left\{e_{k}\right\}_{k=1}^{\infty}$ be an orthonormal basis for $\mathcal{H}$ and consider the frame

$$
\left\{g_{k}\right\}_{k=1}^{\infty}:=\left\{e_{1}, e_{1}, e_{2}, e_{3}, e_{4}, \ldots\right\}
$$

consisting of the orthonormal basis and a single extra element. A natural way to try to remove the redundancy would be to fix a small $\epsilon>0$ and let $\psi_{1}:=e_{1}$ and for $k>1, \psi_{k}:=\frac{1}{2} e_{k-1}+\left(\frac{1}{2}+\epsilon\right) e_{k}$. Then for any finite sequence 


$$
\begin{aligned}
& \left\{c_{k}\right\}_{k=2}^{\infty}, \\
& \left\|\sum_{k=2}^{\infty} c_{k}\left(\left(\frac{1}{2}+\epsilon\right) e_{k}-\psi_{k}\right)\right\|^{2}=\frac{1}{4}\left\|\sum_{k=2}^{\infty} c_{k} e_{k-1}\right\|^{2}=\frac{1}{4} \sum_{k=2}^{\infty}\left|c_{k}\right|^{2} .
\end{aligned}
$$

Observe that $\left\{e_{1}\right\} \cup\left\{\left(\frac{1}{2}+\epsilon\right) e_{k}\right\}_{k=2}^{\infty}$ is a Riesz basis with lower bound $\frac{1}{2}+\epsilon$. Considering $\left\{\psi_{k}\right\}_{k=1}^{\infty}$ as a perturbation of this Riesz basis, it now follows from the results in [5] that $\left\{\psi_{k}\right\}_{k=1}^{\infty}$ is a Riesz basis for $\mathcal{H}$. Note that

$$
\left\|g_{k}-\psi_{k}\right\|=\sqrt{\frac{1}{4}+\left(\frac{1}{2}+\epsilon\right)^{2}} ;
$$

however, this construction does not allow us to obtain $\left\|g_{k}-\psi_{k}\right\| \leq \delta$ when $\delta<2^{-1 / 2} \approx 0.7$. In fact, in order to obtain the result in Theorem 3.4 for smaller values of $\delta$, it would be necessary to consider much more complicated perturbations $\left\{\psi_{k}\right\}_{k=1}^{\infty}$ of $\left\{g_{k}\right\}_{k=1}^{\infty}$, making it highly nontrivial to do this in practice.

Remark 3.7 The question of removal of redundancy is partly motivated by the research topic dynamical sampling, introduced in the papers [3, 1]. One of the key issues in dynamical sampling is the construction of frames as orbits $\left\{T^{k} \varphi\right\}_{k=0}^{\infty}$ of a bounded operator $T: \mathcal{H} \rightarrow \mathcal{H}$, for some $\varphi \in \mathcal{H}$; we encountered such frames already in Example 3.2(ii) \& (iii). Unfortunately it is very difficult to construct such frames, and the only concrete examples available in the literature are indeed Riesz bases [11] and the Carleson frame [1] considered in Example 3.2 (ii). Also, it was proved in [1] that a nearRiesz basis never has this property. This raises the natural question whether a near-Riesz basis can be approximated by a Riesz basis, and hence by an orbit of a bounded operator; Theorem 3.4 confirms that this indeed is possible. We will phrase this consequence of Theorem 3.4 as a separate result, where we index the given near-Riesz basis by $\left\{g_{k}\right\}_{k=0}^{\infty}$ for notational convenience:

Corollary 3.8 Consider any near-Riesz basis $\left\{g_{k}\right\}_{k=0}^{\infty}$. Then, given any $\delta>$ 0 , there exists $\varphi \in \mathcal{H}$ and a bounded operator $T: \mathcal{H} \rightarrow \mathcal{H}$ such that

$$
\left\|g_{k}-T^{k} \varphi\right\| \leq \delta, \forall k \in \mathbb{N}_{0}
$$


The results in Theorem 3.1 and Theorem 3.4 do not cover the standard (regular) redundant Gabor frames and wavelet frames: they consist of vectors with equal norm, and they have infinite excess [4]. Due to the complications discussed in Example 3.1 and the preceding text it seems to be very difficult to answer the question whether all frames indeed can be approximated by a Riesz basis. At least for Gabor frames and wavelet frames we can apply the following adaption of the Feichtinger Theorem (finally proved in one of its equivalent formulations in [14]), showing that any frame which is normbounded below can be approximated by a finite collection of Riesz bases:

Theorem 3.9 Let $\left\{g_{k}\right\}_{k=1}^{\infty}$ be a frame which is norm-bounded below. Then there exists a finite partition $\left\{g_{k}\right\}_{k=1}^{\infty}=\bigcup_{j=1}^{J}\left\{g_{k}\right\}_{k \in I_{j}}$ with the property that for each $\delta>0$ there exist Riesz bases $\left\{\psi_{k}\right\}_{k \in I_{j}}, j=1, \ldots, J$, for $\mathcal{H}$ such that $\left\|g_{k}-\psi_{k}\right\| \leq \delta$ for all $k \in \mathbb{N}$.

Proof. Choose according to the Feichtinger Theorem a finite partition $\left\{g_{k}\right\}_{k=1}^{\infty}=\bigcup_{j=1}^{J}\left\{g_{k}\right\}_{k \in I_{j}}$ such that each sequence $\left\{g_{k}\right\}_{k \in I_{j}}, j=1, \ldots, J$ is a Riesz sequence; using Theorem 2.1 in [11] we can shuffle the elements around to ensure that each of the index sets $I_{j}$ is infinite. Now the result follows directly from Theorem 2.3 (i).

The result in Theorem 3.9 can be formulated as a operator-theoretic result, similarly to Corollary 3.8, we leave the precise formulation to the interested reader.

\section{References}

[1] Aldroubi, A., Cabrelli, C., Molter, U., and Tang, S.: Dynamical sampling. Appl. Appl. Harm. Anal. Appl. 42 no. 3 (2017), 378-401.

[2] Aldroubi, A., Cabrelli, C., Çakmak, A. F., Molter, U., and Petrosyan, A.: Iterative actions of normal operators. J. Funct. Anal. 272 no. 3 (2017), $1121-1146$

[3] Aldroubi, A., Davis, J., and Krishtal, I.: Dynamical sampling: time-space trade-off. Appl. Comp. Harm. Anal. 34 (2013), no. 3, 495--503

[4] Balan, R., Casazza, P., Heil, C. and Landau, Z.: Deficits and excesses of frames. Adv. Comp. Math. 18 (2003), 93-116. 
[5] Casazza, P.G. and Christensen, O.: Frames containing a Riesz basis and preservation of this property under perturbation. SIAM J. Math. Anal. 29 no.1 (1998), 266-278.

[6] Chen, D. Y., Li, L., and Zheng, B. T.: Perturbations of frames. Acta Math. Sinica, English Series, 30 no. 7 (2014), 1089-1108.

[7] Christensen, O.: Frame perturbations. Proc. Amer. Math. Soc. 123 (1995) 1217-1220.

[8] Christensen, O.: Operators with closed range and perturbation of frames for a subspace. Canad. Math. Bull 42 no.1 (1999) 37-45.

[9] Christensen, O.: An introduction to frames and Riesz bases. Second expanded edition. Birkhäuser (2016)

[10] Christensen, O., deFlicht, C. and Lennard, C.: Perturbation of frames for a subspace of a Hilbert space. Rocky Mountain J. Math. 30 no. 4 (2000), 1237-1249.

[11] Christensen, O., and Hasannasab, M.: Frame properties of iterated systems., Appl. Comp. Harm. Anal. 46 (2019), 664-673.

[12] Christensen, O., Hasannasab, M., and Philipp, F: Frame properties of operator orbits. Math. Nach. 293 (2020), 52-66.

[13] Holub, J.: Pre-frame operators, Besselian frames and near-Riesz bases. Proc. Amer. Math. Soc. 122 (1994), 779-785.

[14] Marcus, A., Spielman, D. A., and Srivastava: Interlacing families II: Mixed characteristic polynomials and the Kadison-Singer problem. Ann. of Math. 182 (2015), no. 1, 327-350.

[15] Olevskii, V.: On orthonormal bases and translates. J. Approx. Theory 202 (2016), 1-4.

[16] Olevskii, V.: Completion by perturbations. Preprint, 2020.

[17] Young, R.: An introduction to nonharmonic Fourier series. Academic Press, New York, 1980 (revised first edition 2001). 
Ole Christensen

DTU Compute

Technical University of Denmark

Building 303

2800 Lyngby

Denmark

Email: ochr@dtu.dk

Marzieh Hasannasab

Institut für Mathematik

TU Berlin

Straße des 17. Juni 136

10623 Berlin

Germany

Email: hasannas@math.tu-berlin.de 